\title{
Interfacial Synthesis of Structurally Defined Organic Two-dimensional Materials: Progress and Perspectives
}

\author{
Hafeesudeen Sahabudeen, Renhao Dong, and Xinliang Feng*
}

\begin{abstract}
In the last decade, the development of thin-layer organic 2D materials has attracted considerable attention due to their unique properties arising from 2D planar structures and versatility of organic chemistry. Several synthetic strategies have been developed to synthesize organic 2D materials, such as $2 \mathrm{D}$ polymers, 2D supramolecular polymers as well as single/thin-layer 2D covalent organic frameworks and metal-organic frameworks, either by top-down exfoliation or bottom-up interfacial synthesis methods. Among these, the liquid interface offers a flat and uniform surface for a 2D confinement which renders the preparation of organic 2D materials on a large area under ambient conditions. This review article summarizes the recent developments on the interfacial synthesis of single-layer and few-layer organic 2D materials involving polymerization at the air-water interface by the Langmuir-Blodgett method and at liquid-liquid interfaces. Insights into the perspectives and challenges of synthetic strategies as well as structural characterization are provided for the future development of organic 2D materials.
\end{abstract}

Keywords: 2D polymers $\cdot$ Air-water interface $\cdot$ Liquid-liquid interface $\cdot$ Metal-organic framework $\cdot$ Organic 2D materials

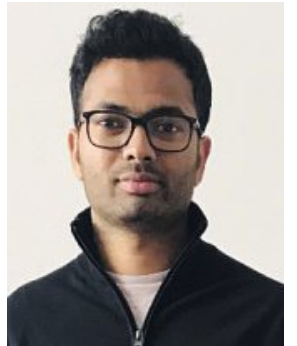

Hafeesudeen Sahabudeen received his master's degree in Nanobiophysics at Technical University of Dresden in 2014. During his MSc studies, he worked on the surface characterization of nanoparticles. In 2015, he joined Prof. Xinliang Feng group to pursue his $\mathrm{PhD}$ degree at the Chair of Molecular Functional Materials at Technical University of Dresden. His current research interests focus on the design and interfacial synthesis of organic 2D materials such as 2D polymers, 2D covalent organic frameworks, and 2D metal-organic frameworks as well as their applications in electronics.

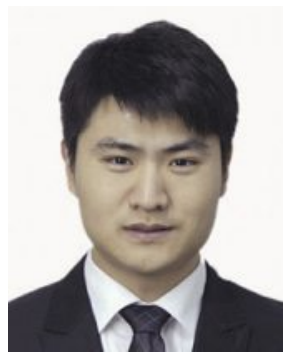

Renhao Dong obtained his BS degree in chemistry from Shandong University in 2008 and completed his PhD studies with a focus on colloid and interface chemistry under the supervision of Prof. Jingcheng Hao at Shandong University (China) in 2013. Then he joined Prof. Klaus Müllen's group as a Postdoctoral Fellow at Max Planck Institute for Polymer Research (Mainz, Germany) in August 2013. In 2015, he joined Prof. Xinliang Feng's group as an Alexander von Humboldt Foundation Fellow at Technical University of Dresden (Germany). Since 2017, he has been a research group leader in the Chair of Molecular Functional Materials at Technical University of Dresden. His current research interests focus on design and synthesis (in solution/at interfaces) of organic 2D materials such as $2 \mathrm{D}$ polymers, $2 \mathrm{D}$ covalent organic frameworks, and 2D metalorganic frameworks as well as their applications in electronics and energy storage/conversion.

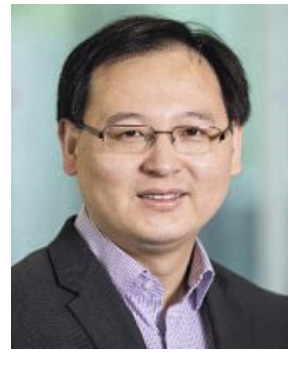

Xinliang Feng is a full professor and has been the head of the Chair of Molecular Functional Materials at Technical University of Dresden since 2014. He received his bachelor's degree in analytic chemistry in 2001 and master's degree in organic chemistry in 2004. Then he joined the Max Planck Institute for Polymer Research for his $\mathrm{PhD}$ studies, where he obtained his $\mathrm{PhD}$ degree in April 2008. In December 2007, he was appointed as a group leader at the Max-Planck Institute for Polymer Research, and in 2012, he became a distinguished group leader at the Max-Planck Institute for Polymer Research. His current scientific interests include organic synthetic methodology, organic synthesis and supramolecular chemistry of $\pi$-conjugated systems, bottom-up synthesis and top-down fabrication of graphene and graphene nanoribbons, 2D polymers and supramolecular polymers, 2D conjugated polymers for opto-electronic applications, energy storage and conversion, new energy devices and technologies.

\section{Introduction}

Organic two-dimensional (2D) materials are generally referred to as laterally infinite, single-layer or few-layer sheets, molecular structurally ordered networks resulting in unique chemical and physical properties. ${ }^{[1,2]}$ The growing interest in organic $2 \mathrm{D}$ materials such as single-layer $2 \mathrm{D}$ polymers ${ }^{[3-5]}$ and $2 \mathrm{D}$ supramolecular polymers, ${ }^{[6,7]}$ single-layer or few-layer $2 \mathrm{D}$ metal-organic frameworks $(\mathrm{MOFs})^{[8]}$ and covalent organic frameworks $(\mathrm{COFs})^{[9-11]}$ is inspired by their crystalline porous structures and intriguing properties as well as their potential applications in electronics and optoelectronics, ${ }^{[12,13]}$ catalysis, ${ }^{[14,15]}$ separation, ${ }^{[16]}$ energy storage ${ }^{[17]}$ and conversion. ${ }^{[18,19]}$ Several synthetic protocols based on 
top-down and bottom-up strategies have been developed to create structurally defined organic 2D materials. Typically, top-down exfoliation of synthetic laminar structures, such as layered $\mathrm{COFs}^{[20]}$ and polymer crystals, has been successfully employed to achieve single-layer or few-layer 2D polymers. Reversible covalent bonds such as boronate ester ${ }^{[10]}$ and imine bonds ${ }^{[21]}$ as well as irreversible condensation reactions like the Knoevenagel reaction ${ }^{[22]}$ and triazine formation ${ }^{[23]}$ have been employed for the construction of layered crystalline COFs. Thus, this strategy allows the design of precisely controllable structures since their chemical and physical properties can be easily tuned through the selection of the building blocks and linkage chemistries. Nevertheless, problems still arise from structural defects due to high or low rates of reversibility of bond formation and instability of exfoliated sheets. On the other hand, the synthesis of 2D polymer crystals is mainly based on topochemical polymerization. For example, anthracenebased monomers are pre-organized into lamellar crystals and subsequently polymerized through a photoreaction to achieve layered single crystals of $2 \mathrm{D}$ polymers, which are then exfoliated into thin nanosheets. ${ }^{[24]}$ However, the lateral sizes of the obtained 2D polymer sheets are ultimately limited by the size of the layered crystals and the precise thickness control of the exfoliated sheets down to single-layer level remains challenging. ${ }^{[25]}$

In the case of 'bottom-up' strategies, the interfacial synthesis method is an efficient way to prepare single- or few-layer organic $2 \mathrm{D}$ materials ${ }^{[26]}$ where it typically involves reaction at the interface between air-water (or gas-liquid), [27,28] liquid-liquid,[6,29] liquid-solid, and gas-solid (or vacuum-solid). ${ }^{[30]}$ For instance, early in 2007, on-surface synthesis rendered the formation of 2D poly(m-phenylene), ${ }^{[30]}$ and covalent assemblies of porphyrin and thiophene monomers. ${ }^{[31]}$ In a typical procedure, monomers are deposited via sublimation or drop-casted onto highly oriented pyrolytic graphite or metal surfaces and then polymerized at elevated temperature under ultra-high vacuum (UHV) conditions. There, non-covalent interaction mediated by metal substrates and arylaryl coupling reaction as well as dynamic covalent reactions such as boroxine, ${ }^{[32]}$ imine ${ }^{[33]}$ bond formation, etc., can be employed to construct 2D networks on solid substrates. Since most of the reactions are performed on the flat surfaces, the generated 2D nanostructures can be directly visualized under high-resolution atomic force microscopy (AFM) and scanning tunneling microscopy (STM) with atomic precision. However, this strategy only produces 2D networks with small crystalline domain sizes (tens of nanometers) and high defect density, which are most likely induced by the low mobility of monomers and reactivity at solidvacuum interfaces. Moreover, a transfer of resultant 2D networks from the metal substrate is quite challenging which hinders their potential applications.

In order to overcome the limitations associated with the exfoliation and on-surface synthesis methods, air-water and liquidliquid interface assisted syntheses have been employed for the preparation of 2D polymers, 2D supramolecular polymers as well as single-layer or few-layer 2D MOFs and 2D COFs. The interface itself holds unique features which are ideal to construct the organic 2D nanostructures: 1) The water surface is smooth and uncorrugated with low surface roughness up to $3 \AA$; such smooth surfaces provide a unique flat environment to control the dynamic interaction (chemical reaction) within the interfacial region. ${ }^{[34]} 2$ ) The molecular orientation of anchoring molecules is of significant importance which influences the dynamics of a chemical reaction and determines the formation of final 2D structures with molecular ordering at the interface. ${ }^{[7,35]}$ In this case, the molecular orientation can be tailored by the solvent/monomer or monomer/monomer intermolecular forces, which typically includes electrostatic force, hydrogen-bonding, hydrophilic solvation, and hydrophobic effects.[36] 3) The water surface offers lateral mobility of molecules and confines the transport processes into two dimensions, which results in long-range ordered 2D structures. Therefore, the $2 \mathrm{D}$ confined assembly at the air-water interface has great potential to mimic various solid structures of desired properties. 4) The interfacial synthesis method allows for the preparation of largearea organic 2D structures with tunable thickness ranging from single-layer to multilayers, which can be readily transferred onto an arbitrary substrate for further characterizations and device fabrications.

In this review article, we will summarize the recent development of interfacial synthesis methodologies and mainly focus on the air-water and liquid-liquid interface strategies for the synthesis of 2D polymers, single-layer or few-layer 2D MOFs and multilayer 2D COFs with representative work from few selective examples.

\section{Synthesis at the Air-Water Interface}

The water surface provides a unique environment to study the orientation of molecules in a confined space and facilitates polymerization along the 2D direction. The experimental and theoretical concepts of monolayer investigation at the air-water interface were first systematically analyzed by Langmuir in 1917. ${ }^{[37]}$ Later, in the early 1930s, Gee and Rideal reported stabilized monolayers through oxidation mechanism of $\beta$-elaeostearin and this was the first report towards the polymerization of the crosslinked monolayer at the air-water interface. ${ }^{[38]}$ In the meantime, Blodgett developed a new transfer method where multilayers of the calcium salt of stearic acid from the air-water interface were transferred onto various solid substrates, which paved the way for the Langmuir-Blodgett (LB) technique. ${ }^{[39]}$ In a typical synthesis procedure (Fig. 1a), amphiphilic monomers are spread on the water surface in a Langmuir trough and are mechanically compressed into a monomer monolayer. Upon compression, they form a densely packed monomer monolayer film where the monomer reactive sites are in close contact with each other. At this stage, polymerization is triggered by light irradiation or by diffusion of reagents from the sub-phase to the reactive sites of the monomers. The LB technique enables the conversion of simple molecules into layered structures and offers the possibility of control over the chemistry of sub-phase and thermodynamic variables such as surface pressure, $\mathrm{pH}$, etc. One of the attractive features of this technique is that single-layer 2D polymers, 2D supramolecular polymers, and MOF films can be synthesized with lateral size limited only along with the extension of water surface. However, characterization of the monolayer organic films synthesized at the interface has always been a challenge due to their high sensitivity to the electron irradiation of transmission electron microscopy (TEM) and structural distortion such as folding and tearing of the sheets during the transfer process. The recent development of microscopic imaging techniques in the past decade with regard to STM, TEM, and AFM have enabled the possibility to directly elucidate the organic 2D material structure at the molecular level. This technological advancement triggered the development of a different class of organic 2D materials.

The interfacial synthesis based on metal coordination chemistry is the most straightforward approach to obtain single-layer 2D structures. In 2002, a 2D iron-nickel cyanide-bridged network based on metal coordination assembly was reported by linking amphiphilic pentacyanoferrate (III) and nickel ions using an LB trough. ${ }^{[40]}$ In a typical procedure, the pentacyanoferrate (III) in chloroform solution was spread on the water surface in an LB trough and metal ions such as $\mathrm{Ni}^{2+}$ were injected into the water phase. The metal ions in the sub-phase diffused to the water surface and reacted with pentacyanoferrate (III) to form a monolayer MOF structure. By this way, a free-standing monolayer MOF was achieved in a large area which could be readily transferred onto solid/holey substrates for further characterization. Nevertheless, the crystallinity of the monolayer MOF structure was not dem- 

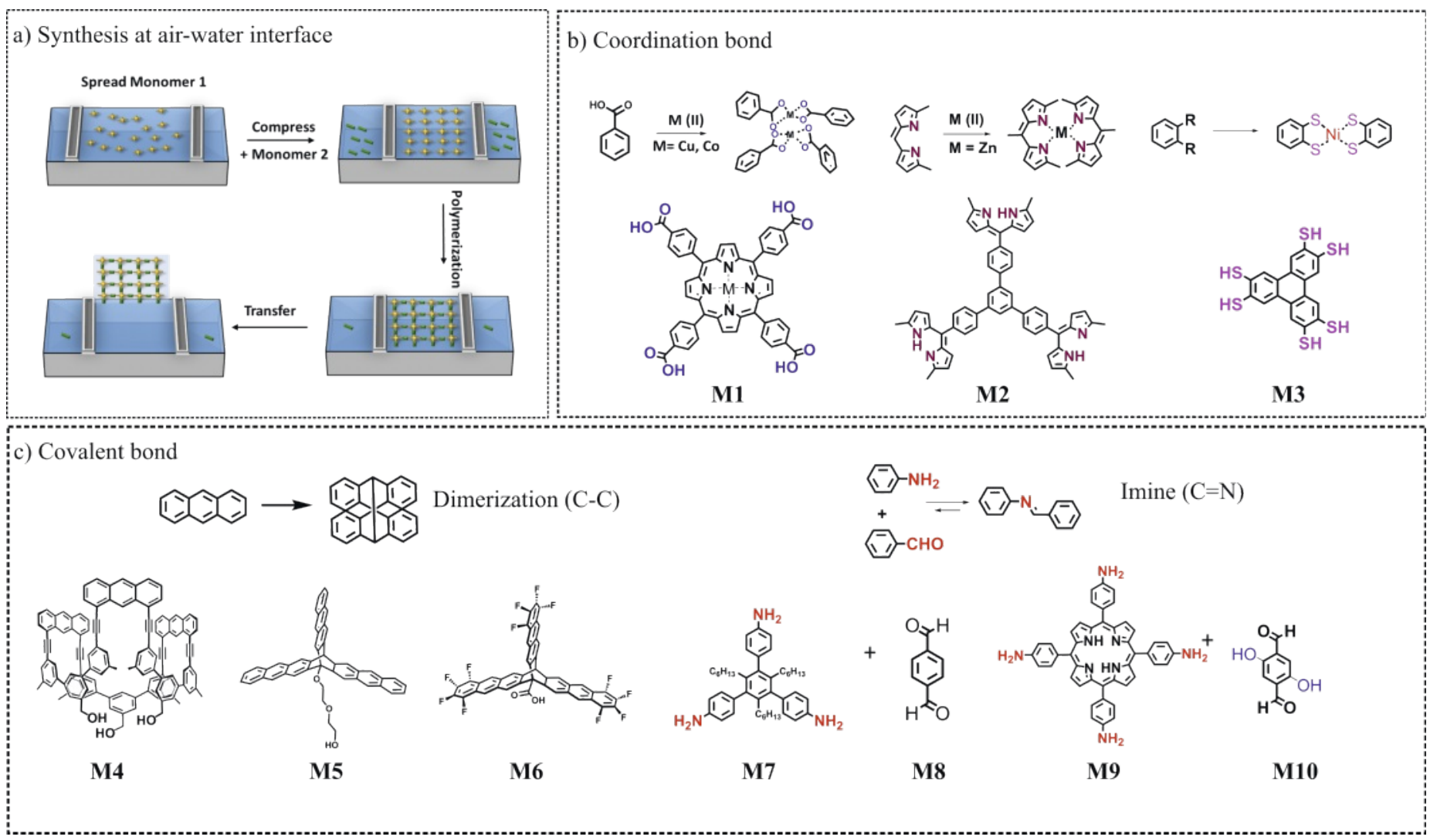

Fig. 1. a) Schematic representation of the synthesis of 2D polymers at the air-water interface by the Langmuir-Blodgett technique. Representative monomers and reaction scheme for the synthesis of b) metal coordination 2D networks, c) 2D polymers based on photodimerization and imine chemistry.

onstrated at that time. As a step further, in 2010, the construction of porphyrin-based MOF nanosheets was investigated by in situ synchrotron grazing incidence X-ray diffraction (GIXRD) at the air-water interface. ${ }^{[35]}$ The synthesis of monolayer $2 \mathrm{D}$ MOF was achieved through the complexation of the $\mathrm{Cu}^{2+}$ and $\mathrm{COO}^{-}$group of the porphyrin units (M1, Fig. 1b). The 4-fold symmetry of the porphyrin ligand facilitated the construction of a grid structure and the stacked MOF film was achieved through the layer-by-layer growth technique. The periodicity of such stacked MOF structures was demonstrated by synchrotron X-ray diffraction (XRD), which showed crystalline ordering in both in-plane and out-of-plane directions with an average crystal domain size of $\sim 20 \mathrm{~nm}$. Many efforts have been dedicated aiming to prepare monolayer MOF with high molecular structural periodicity. However, to disclose the internal structure of a monolayer MOF sheet itself has been a big challenge. In 2015, we demonstrated the synthesis of a largearea (square centimeters), free-standing monolayer 2D MOF (0.7 $\mathrm{nm}$ in thickness, also named as the 2D supramolecular polymer in this work) comprising triphenylene-fused nickel bis(dithiolene) complexes (M3) at the air-water interface. ${ }^{[28]}$ To minimize the effect of electron damage on monolayer MOF structure, the internal periodicity was elucidated by Cryo-TEM at $-175^{\circ} \mathrm{C}$ which exhibited the crystalline diffraction pattern of a hexagonal ordered network (Fig. 2a). The spectroscopic characterizations including XPS and EDX suggested the efficient complexation between $\mathrm{Ni}^{2+}$ ions and sulfur groups in the MOF structure with a ratio of metal $(\mathrm{Ni})$ to sulfur in the range of 1:4.8. The achieved singlelayer MOF film was then immobilized onto the electrode surface which functioned as an active electrode material for electrocatalytic hydrogen generation reaction. It should be mentioned that significant progress has been made over the last several years for the synthesis of monolayer MOFs by employing N, O, or S orthodisubstituted benzene ${ }^{[41,42]}$ or terpyridine ${ }^{[7]}$ or dipyrrin ${ }^{[43]}$ triphenylene ligands. ${ }^{[28]}$
Besides the exploration of 2D MOF structures, 2D polymers formed at the air-water interface have also attracted great attention in recent years. The versatility of organic reactions between molecular building blocks can provide crystalline 2D structures with tailored functionalities, which makes them suitable for diverse applications. In 2014, Schlüter and his coworkers synthesized mechanically stable $\mathrm{C}-\mathrm{C}$ coupled monolayer $2 \mathrm{D}$ polymers through UV-induced photo-dimerization reaction at the air-water interface. ${ }^{[44]}$ Specifically, photoactive anthracene monomers (M4) were pre-organized to form a monomer monolayer at the air-water interface. Upon photo-irradiation, a [4+2]-cycloaddition reaction was triggered between neighboring monomers which resulted in free-standing monolayer covalent sheets. Though the polymerization was confirmed by tip-enhanced Raman spectroscopy (TERS), the structural periodicity was not confirmed due to structural flexibility of the monomers. In 2015, photodimerization of a shape-persistent antrip monomer (M5, Fig. 1c) at the air-water interface was reported for the synthesis of a monolayer $2 \mathrm{D}$ polymer. ${ }^{[45]}$ The obtained free-standing $2 \mathrm{D}$ polymer was structurally characterized by STM, which showed a honeycomb periodic lattice structure with ultra-pore density of $\sim 6.8 \times 10^{13}$ pores $/ \mathrm{cm}^{2}$. More recently, the same group reported the structural confirmation of a fluorinated anthracene (M6) based 2D polymer using the nc-AFM technique. ${ }^{[46]}$ Remarkably, the 2D polymer formed at the air-water interface was transferred onto a highly oriented pyrolytic graphite (HOPG) substrate and subsequently imaged by the nc-AFM technique which revealed monodisperse pores (in the range of $\sim 1.8$ to $2.1 \mathrm{~nm}$ ) in the monolayer, which extended over the entire area of $300 \times 300 \mathrm{~nm}^{2}$.

In addition to the photodimerization reaction based on carboncarbon bonds, reversible dynamic covalent reactions such as the Schiff-base reaction are also attractive chemistry methodologies for the construction of robust $2 \mathrm{D}$ polymers at the air-water interface. The self-correction mechanism associated with the dynamic 


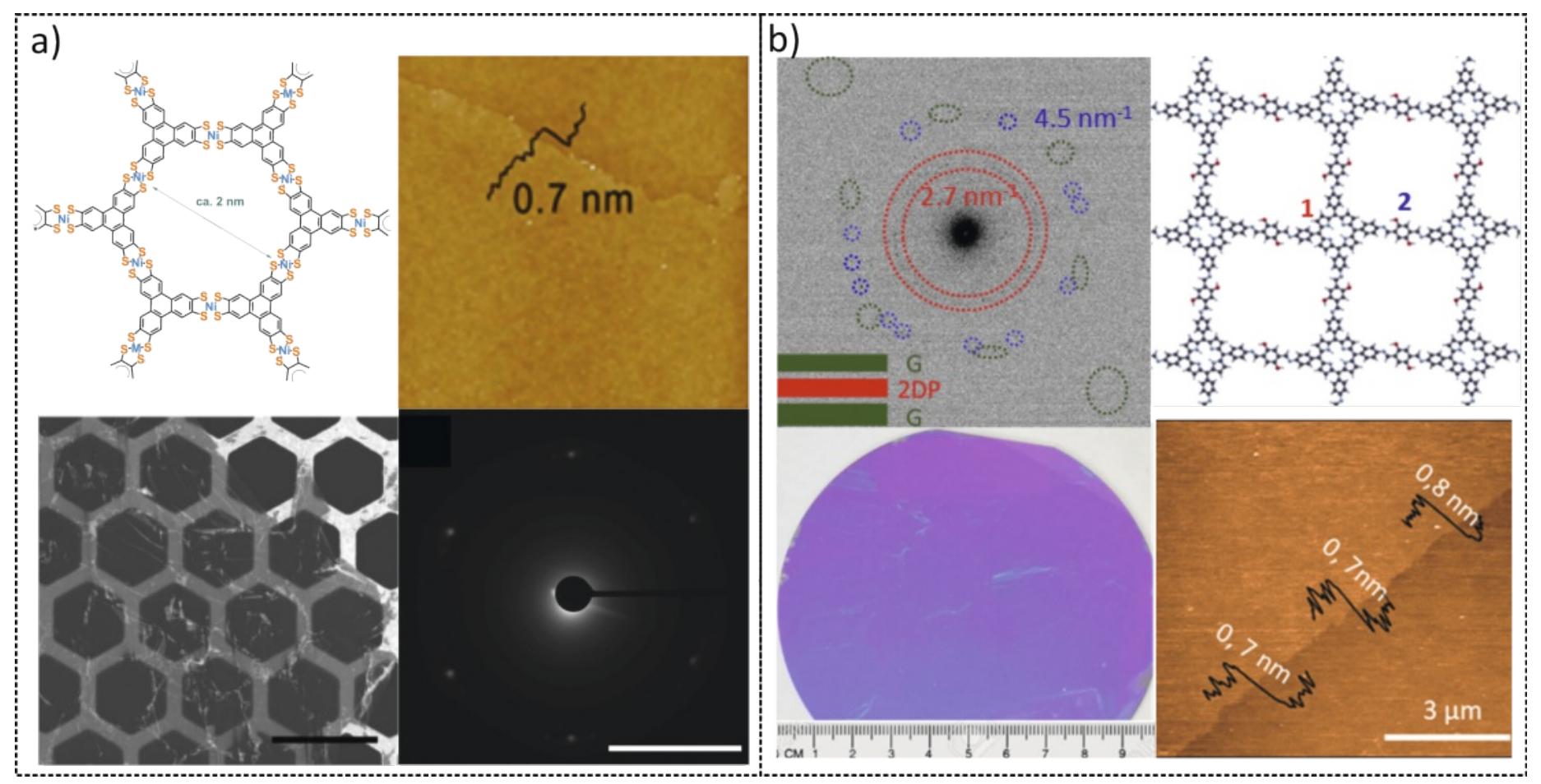

Fig. 2. a) Selected example of a single-layer 2D MOF film synthesized at the air-water interface based on triphenylenehexathiol (THT) monomer. The synthesized monolayers are free-standing and exhibit hexagonal crystalline structure as confirmed by the SAED analysis. Adapted with permission from ref. [28]. b) Selected examples of conjugated 2D polymers based on imine chemistry. The crystallinity of the wafer-sized monolayer 2D polyimine was demonstrated by the SAED analysis using a graphene sandwich technique. Adapted with permission from ref. [27]. Copyright 2016 Nature Publishing Group and 2015 John Wiley \& Sons, Inc, respectively.

nature of imine chemistry offers a promising protocol for the synthesis of 2D polymers at the water-containing interfaces. In 2016, we reported a wafer-sized crystalline $2 \mathrm{D}$ polymer through the reaction between 5,10,15,20-tetrakis(4-aminophenyl)-21 $\mathrm{H,23H}$ porphyrin (M8) and 2,5-dihydroxyterephthalaldehyde (M9) at the air-water interface ${ }^{[27]}$ (Fig. 2b). The synthetic method involves spreading a submonolayer of porphyrin on the water surface in an LB trough. After the close packing of the monomers into a dense film upon compressing to $10 \mathrm{mN} \mathrm{m}^{-1}$, water-soluble aldehyde monomers were injected in the sub-phase and then diffused to the air-water interface where $2 \mathrm{D}$ polymerization was triggered by the formation of imine bonds between amine and aldehyde groups. The resultant monolayer $2 \mathrm{D}$ polymer possessed a thickness of $\sim 0.7 \mathrm{~nm}$ with a lateral size of a 4-inch wafer and exhibited a Young's modulus of $267 \pm 30 \mathrm{GPa}$. The internal structure of the monolayer 2D polymer was investigated by electron diffraction performed in a TEM. However, due to the high electron beam sensitivity, the free-standing 2D polymer deteriorated rapidly and no diffraction pattern could be recorded even under cryogenic conditions. To combat the negative effects of electron radiation, the low-dose technique combined with a novel graphene encapsulation technique was applied to enhance the radiation resistance of the monolayer 2D polymer. In this way, monolayer 2D polymer was encapsulated between two graphene layers and subsequent electron diffraction measurement clearly showed the polycrystalline feature of this $2 \mathrm{D}$ polymer. This technique can be well adapted to characterize other single-layer organic 2D materials. Furthermore, the achieved 2D polymer could function as an active semiconducting layer in an organic thin film transistor (OTFT), whereas the $2 \mathrm{D}$ polymer derivative employing cobalt-porphyrin monomer could efficiently catalyze hydrogen generation from water.

\section{Synthesis at the Liquid-Liquid Interface}

Liquid-liquid interfaces are formed between two immiscible liquids, i.e. an organic-aqueous phase and provide confined $2 \mathrm{D}$ space at phase boundaries for the growth of organic 2D materials. The liquid-liquid interface usually has a thickness of a few nanometers, which acts as a template to confine monomers and their polymerization into single-layer or few-layer organic 2D structures. In this respect, few-layer COF and MOF films have been successfully synthesized via diffusion of monomers or ligands and salts at the liquid-liquid interface. ${ }^{[29,48,49]}$ For instance, in 2015, Nishihara and co-workers reported multilayer 2D MOF sheets through complexation of trifunctional dipyrrin ligand (M2) and zinc(II) acetate at the dichloromethane-water interface. ${ }^{[43]}$ The thickness of the MOF film could be tuned from 6 to $800 \mathrm{~nm}$. Recently, we reported the synthesis of semiconducting, $\pi-d$ conjugated $2 \mathrm{D}$ MOF films at a $\mathrm{CH}_{3} \mathrm{Cl}$-water interface. ${ }^{[49]}$ The liquid-liquid interface was applied by dissolving 2,3,6,7,10,11-triphenylenehexathiol (THT) monomer in water along with $0.01 \mathrm{M} \mathrm{NH}_{4} \mathrm{OH}$ (top phase) and iron acetylacetonate in $\mathrm{CH}_{3} \mathrm{Cl}$ (bottom phase). The thickness of the resultant crystalline 2D MOF film could be tuned from $20 \mathrm{~nm}$ to a few hundreds of nanometers. The obtained $\pi-d$ conjugated 2D MOF films exhibited band-like charge carrier transport with record mobility of $\sim 220 \mathrm{~cm}^{2} / \mathrm{Vs}$ among all reported conducting MOF materials.

Clearly, the synthetic strategy using the liquid-liquid interface can be also employed to prepare multilayer 2D COF or 2D polymer thin films. In 2016, we employed the liquid-liquid interface approach to synthesize crystalline multilayer 2D polymers based on the Schiff-base reaction (Fig. 3). ${ }^{[27]}$ The interface was created by dissolving 2,5-dihydroxyterephthalaldehyde in aqueous solution (top-phase) and 10,15,20-tetrakis(4aminophenyl) $21 H, 23 H$-porphyrin-Co(II) in chloroform (bottom-phase). The interfacial reaction was kept undisturbed for 10 days, which yielded a multilayer film with a uniform thickness of $\sim 20 \mathrm{~nm}$ and exhibited high crystallinity with a square structure based on HR-TEM and SAED patterns. Furthermore, Raman spectroscopy and XPS confirmed the efficient formation of imine bond in multilayer film synthesized at the liquid-liquid interface. 


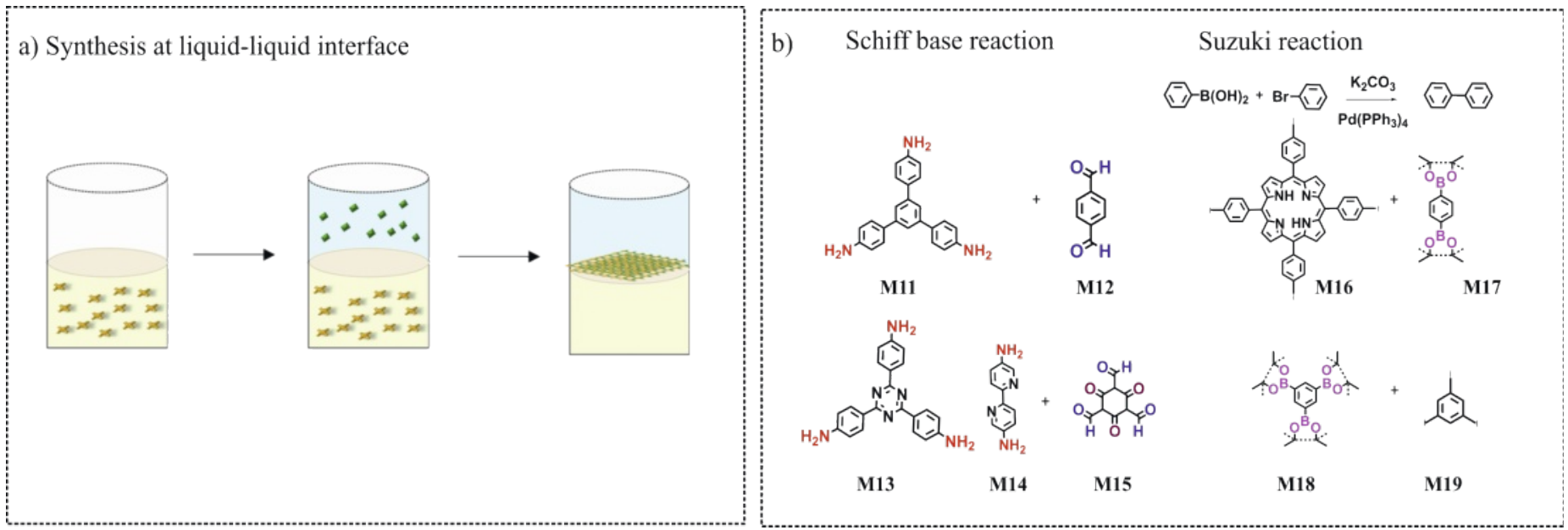

Fig. 3. a) Schematic representation of the synthesis of 2DP at the liquid-liquid interface. b) Representative monomers used for the synthesis of 2DPs based on imine and Suzuki polymerization at the liquid-liquid interface.

In 2017, Dichtel and coworkers employed Lewis acid $\mathrm{Sc}(\mathrm{OTf})_{3}$ as an active catalyst to synthesize polyimine COF films at the liquid-liquid interface. ${ }^{[47]}$ The Lewis acid catalyst is water tolerant and can mediate imine formation, thus enabling the enhancement of the crystallinity of the resultant imine frameworks. In this work, the interface consists of 1,3,5-tris(4-aminophenyl) benzene (M11) in the aqueous phase and terephthalaldehyde (M12) dissolved in 1,4 dioxane/mesitylene containing $\mathrm{Sc}(\mathrm{OTf})_{3}$. The reaction was kept undisturbed for $72 \mathrm{~h}$ yielding a crystalline film, where the thickness could be tuned from $2.5 \mathrm{~nm}$ to $100 \mu \mathrm{m}$ by varying the concentration of precursor monomers. Furthermore, the obtained 2D COF films as nanofiltration (NF) membranes exhibited a high rejection of rhodamine dye as a model organic pollutant. In another example, a salt-mediated technique was demonstrated in 2017 to synthesize crystalline imine-based 2D COF thin film (M13, M14, M15) at the dichloromethane-water interface under ambient conditions ${ }^{[51]}$ (Fig. 4a). In a typical synthetic procedure, amine- $p$-toluene sulfonic acid (PTSA) was used to replace free amine in the aqueous phase, where $\mathrm{H}$ bonding within PTSA-amine salt slowed down the diffusion rate of precursor through the interface which enhanced the reaction towards the thermodynamically controlled crystallization. This strategy has been successfully extended to synthesize 2D COF thin films with different pore sizes as shown in Fig. 4 a
In addition to the dynamic covalent chemistry, irreversible reactions such as Suzuki polymerization was also explored recently at the liquid-liquid interface for the synthesis of 2D polymers ${ }^{[50]}$ (Fig. 4b). Specifically, water-insoluble components including aryl boronic ester (M17, M18), aryl halide (M16, M19), and palladium catalyst were dissolved in toluene phase while the aqueous phase comprised of inorganic base $\mathrm{K}_{2} \mathrm{CO}_{3}$. As described in this work, the interface promoted the transmetalation step by facilitating the interaction of organic components with the inorganic base and the reaction was kept under low temperature conditions yielding a multilayered film after 4 weeks. Nevertheless, the crystalline domain size was too small to evaluate the internal periodicity of such 2D polymer films. In another example, in 2017, Nishihara and coworkers reported the synthesis of graphdiyne sheets via homocoupling polymerization at the liquid-liquid interface. ${ }^{[51]}$ The interface was formed by dichloromethane solution of hexaethynylbenzene (HEB) and the aqueous solution of a copper catalyst. Multilayer graphdiyne sheets with a thickness of $24 \mathrm{~nm}$ were obtained which exhibited in-plane periodicity matching ABC stacking as demonstrated by SAED measurements. Although this strategy is appealing towards the synthesis of few-layer to multi-layer 2D polymer films under mild synthetic conditions, the structure elucidation concerning the nature of grains, defects and grain boundaries of such crystalline materials remain unexplored.
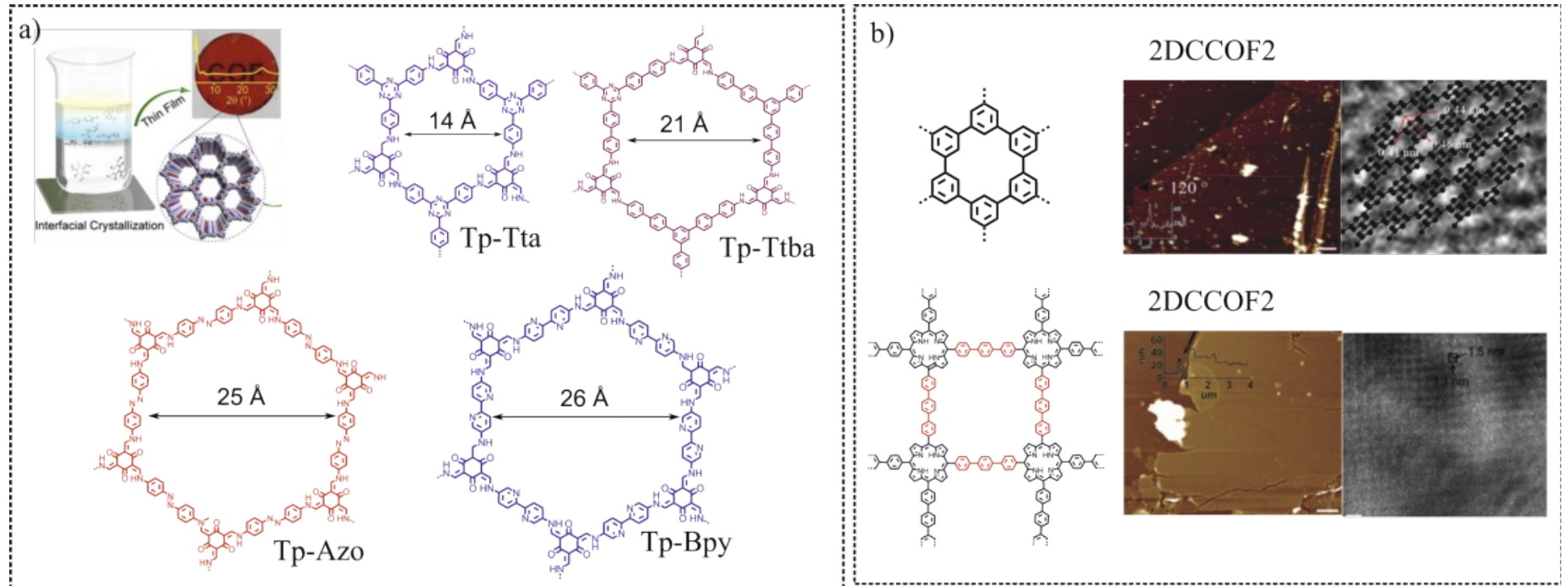

Fig. 4. a) Selected examples of multilayer COF thin films based on imine chemistry at the liquid-liquid interface. Adapted with permission from ref. [29]. b) Selected examples based on the synthesis of COF films linked by C-C bonds. Adapted with permission from ref. [50]. Copyrights 2017 American Chemical Society, and 2019 John Wiley \& Sons, Inc, respectively. 


\section{Challenges and Future Perspectives}

Bottom-up synthesis is a comparatively effective approach for the controlled synthesis of single-layer or few-layer organic 2D materials with finely tuned lateral size and thickness at the molecular level. Despite the preliminary success some critical issues have to be further addressed, such as monomer design, synthetic methodologies as well as structural characterizations.

(i) The interfacial strategy is advantageous for the synthesis of large-area organic 2D materials. However, the issues concerning structural periodicity such as controlling crystalline phases, grain size, grain boundaries, and edge structures of the synthetic organic 2D materials have not been explored so far. Those structural features strongly depend on the physical and chemical properties of organic 2D materials. In this aspect, unique analytical methods have to be established to determine their structures at the interface and successful realization of the (polymerization) reaction at the interfaces. The HR-TEM imaging technique is certainly highly valuable to elucidate the molecular structure of the achieved organic 2D structures, whereas AFM and STM seem to be more challenging thus far due to the possible introduction of contaminations and mechanical instability of monolayers during the transfer process.

(ii) The interfacial strategies have their intrinsic limitations in terms of reaction conditions like temperature, $\mathrm{pH}$, etc., which limit the application scope of some chemical reactions. So far irreversible condensation reactions (photopolymerization, Suzuki and Glaser coupling) and dynamic covalent reactions (Schiff-base reaction) have been explored to synthesize crystalline $2 \mathrm{D}$ polymer nanosheets while other linkage reaction methodologies remain to be explored at the air-water and liquid-liquid interfaces. Furthermore, deeper understanding of reaction mechanism like molecular interaction at the interface or diffusion process of precursor monomers through the interface will further improve the controlled synthesis with high structural periodicity.

(iii) The amount of material used at the interface to synthesize a single layer of organic 2D materials is typically in the range of micrograms which makes it more challenging for further characterization, especially structural analysis at the molecular level. Spectroscopic methods such as fluorescence, IR, Raman spectroscopy and TERS have been employed to confirm bond formation. On the other hand, the internal structural determination of ultrathin organic 2D materials at the molecular level remains a big challenge. Since organic 2D materials are not stable under electron irradiation, to characterize their structural periodicity and crystallinity under TEM conditions is still a non-trivial task. In this case, the graphene sandwich technique, i.e. graphene/2DM/ graphene sandwich structure could be utilized to characterize the crystallinity of the beam-sensitive 2D materials.[27]

(iv) The pre-organization of monomers is a critical step which would control the topology and crystallinity of final organic 2D materials synthesized at the interfaces. In this respect, templateassisted synthesis strategies such as the surfactant bilayer templating method provide the opportunity to fine control the size and morphology of the resulting 2D nanostructures. ${ }^{[52,53]}$ Surfactants can self-organize into stable bilayer structures in aqueous solution and such bilayer surface can be positively or negatively charged or modified with various functional groups, which provide the possibility to guide the growth of ultrathin nanosheets. In 2016, our group demonstrated the bottom-up synthesis of conducting polymer nanosheets such as polypyrrole (mPPy) and polyaniline (PANI) via surfactant bilayer templating. ${ }^{[54]}$ For instance, the mPPy nanosheets with controlled pore sizes ranging from 6.8 to $13.6 \mathrm{~nm}$ and thicknesses of $25-30 \mathrm{~nm}$ were synthesized via synergic self-assembly of amphiphilic aliphatic amine (octadecyl amine, OTA) and polystyrene-b-poly (ethylene oxide) (PS-bPEO) block copolymers (BCPs). Owing to the great advantages in the controllable preparation, precise thickness control, and easy removal without residue, such $2 \mathrm{D}$ bilayer structures provide an ideal platform for the construction of 2D mesoscale-ordered superstructures. Such soft-template-assisted synthesis approaches can be certainly extended to synthesize other types of organic 2D materials such as $2 \mathrm{D}$ polymers at the different interfaces.

(v) The interfacial synthesis approach allows for the preparation of large-area, free-standing organic $2 \mathrm{D}$ materials ranging from single-layer to multilayers which can be transferred to any substrate for device fabrication. With the integration of different functional groups and easy transfer procedures, organic 2D materials will find a wide range of applications in optoelectronics, electronics, sensing, separation, energy storage and conversion. Nevertheless, the control over structural manipulation at the atomic and molecular level is required in order to achieve superior device performance of an expected application. Thus, reliable structure-property relationships have to be established, where the defects, grain boundaries as well as the impurities would exert a large effect on the properties especially related to applications in electronics, spintronics, etc.

\section{Acknowledgments}

X.F. thanks his group members in the fields of interfacial synthesis and 2D polymers, particularly, Tao Zhang, Kejun Liu, Sangwook Park and Zhiyong Wang for valuable discussions. This work is financially supported from EU Graphene Flagship, ERC T2DCP, Coordination Networks: Building Blocks for Functional Systems (SPP 1928, COORNET), H2020-MSCA-ITN (ULTIMATE), as well as the German Science Council and Center of Advancing Electronics Dresden (cfaed).

Received: May 23, 2019

[1] X. Feng, A. D. Schlüter, Angew. Chem. Int. Ed. 2018, 57, 13748.

[2] R. Dong, T. Zhang, X. Feng, Chem. Rev. 2018, 118, 6189.

[3] J. Sakamoto, J. van Heijst, O. Lukin, A. D. Schlüter, Angew. Chem. Int. Ed. 2009, 48, 1030 .

[4] P. Payamyar, B. T. King, H. C. Öttinger, A. D. Schlüter, Chem. Commun. $\mathbf{2 0 1 5}, 52,18$.

[5] J. W. Colson, W. R. Dichtel, Nat. Chem. 2013, 5, 453.

[6] M. Pfeffermann, R. Dong, R. Graf, W. Zajaczkowski, T. Gorelik, W. Pisula, A. Narita, K. Müllen, X. Feng, J. Am. Chem. Soc. 2015, 137, 14525.

[7] T. Bauer, Z. Zheng, A. Renn, R. Enning, A. Stemmer, J. Sakamoto, A. D. Schlüter, Angew. Chem. Int. Ed. 2011, 50, 7879.

[8] M. Zhao, Q. Lu, Q. Ma, H. Zhang, Small Meth. 2017, 1, 1600030.

[9] Y. Jin, Y. Hu, W. Zhang, Nat. Rev. Chem. 2017, 1, 0056.

[10] C. S. Diercks, O. M. Yaghi, Science 2017, 355, eaal1585.

[11] N. Huang, P. Wang, D. Jiang, Nat. Rev. Mater. 2016, 1, 16068.

[12] G. Wu, J. Huang, Y. Zang, J. He, G. Xu, J. Am. Chem. Soc. 2017, 139, 1360

[13] S. Wan, J. Guo, J. Kim, H. Ihee, D. Jiang, Angew. Chem. Int. Ed. 2008, 47, 8826.

[14] J. Lee, O. K. Farha, J. Roberts, K. A. Scheidt, S. T. Nguyen, J. T. Hupp, Chem. Soc. Rev. 2009, 38, 1450 .

[15] S.-Y. Ding, J. Gao, Q. Wang, Y. Zhang, W.-G. Song, C.-Y. Su, W. Wang, J. Am. Chem. Soc. 2011, 133, 19816.

[16] Y. Peng, Y. Li, Y. Ban, H. Jin, W. Jiao, X. Liu, W. Yang, Science 2014, 346, 1356.

[17] D. Sheberla, J. C. Bachman, J. S. Elias, C.-J. Sun, Y. Shao-Horn, M. Dincâ, Nat. Mater. 2017, 16, 220.

[18] R. Dong, Z. Zheng, D. C. Tranca, J. Zhang, N. Chandrasekhar, S. Liu, X Zhuang, G. Seifert, X. Feng, Chem. Eur. J. 2017, 23, 2255.

[19] V. S. Vyas, F. Haase, L. Stegbauer, G. Savasci, F. Podjaski, C. Ochsenfeld, B. V. Lotsch, Nat. Comm. 2015, 6, 8508.

[20] A. P. Côté, A. I. Benin, N. W. Ockwig, M. O’Keeffe, A. J. Matzger, O. M. Yaghi, Science 2005, 310, 1166.

[21] J. L. Segura, M. J. Mancheño, F. Zamora, Chem. Soc. Rev. 2016, 45, 5635.

[22] X. Zhuang, W. Zhao, F. Zhang, Y. Cao, F. Liu, S. Bi, X. Feng, Polym. Chem. 2016, 7, 4176.

[23] P. Kuhn, M. Antonietti, A. Thomas, Angew. Chem. Int. Ed. 2008, 47, 3450.

[24] M. J. Kory, M. Wörle, T. Weber, P. Payamyar, S. W. van de Poll, J. Dshemuchadse, N. Trapp, A. D. Schlüter, Nat. Chem. 2014, 6, 779.

[25] W. Wang, A. D. Schlüter, Macromol. Rapid Comm. 2019, 40, 1800719.

[26] L. Wang, H. Sahabudeen, T. Zhang, R. Dong, npj 2D Mater. Appl. 2018, 2, 26.

[27] H. Sahabudeen, H. Qi, B. A. Glatz, D. Tranca, R. Dong, Y. Hou, T. Zhang, C. Kuttner, T. Lehnert, G. Seifert, U. Kaiser, A. Fery, Z. Zheng, X. Feng, Nat. Comm. 2016, 7, 13461. 
[28] R. Dong, M. Pfeffermann, H. Liang, Z. Zheng, X. Zhu, J. Zhang, X. Feng, Angew. Chem. Int. Ed. 2015, 54, 12058.

[29] K. Dey, M. Pal, K. C. Rout, S. Kunjattu H, A. Das, R. Mukherjee, U. K. Kharul, R. Banerjee, J. Am. Chem. Soc. 2017, 139, 13083.

[30] M. Bieri, M. Treier, J. Cai, K. Aït-Mansour, P. Ruffieux, O. Gröning, P. Gröning, M. Kastler, R. Rieger, X. Feng, K. Müllen, R. Fasel, Chem. Commun. 2009, 6919.

[31] L. Grill, M. Dyer, L. Lafferentz, M. Persson, M. V. Peters, S. Hecht, Nat. Nanotech. 2007, 2, 687.

[32] C.-Z. Guan, D. Wang, L.-J. Wan, Chem. Commun. 2012, 48, 2943.

[33] I. Janica, V. Patroniak, P. Samori, A. Ciesielski, Chem. Asian J. 2018, 13, 465.

[34] A. Braslau, M. Deutsch, P. S. Pershan, A. H. Weiss, J. Als-Nielsen, J. Bohr, Phys. Rev. Lett. 1985, 54, 114.

[35] R. Makiura, S. Motoyama, Y. Umemura, H. Yamanaka, O. Sakata, H. Kitagawa, Nat. Mater. 2010, 9, 565.

[36] D. C. Rideout, R. Breslow, J. Am. Chem. Soc. 1980, 102, 7816.

[37] D. R. Talham, Chem. Rev. 2004, 104, 5479.

[38] G. Gee, E. K. Rideal, Proc. Roy. Soc. Lon. A 1935, 153, 116

[39] K. B. Blodgett, J. Am. Chem. Soc. 1935, 57, 1007.

[40] J. T. Culp, J.-H. Park, D. Stratakis, M. W. Meisel, D. R. Talham, J. Am. Chem. Soc. 2002, 124, 10083.

[41] T. Kambe, R. Sakamoto, K. Hoshiko, K. Takada, M. Miyachi, J.-H. Ryu, S. Sasaki, J. Kim, K. Nakazato, M. Takata, H. Nishihara, J. Am. Chem. Soc. 2013, 135, 2462.

[42] N. Lahiri, N. Lotfizadeh, R. Tsuchikawa, V. V. Deshpande, J. Louie, J. Am. Chem. Soc. 2017, 139, 19.
[43] R. Sakamoto, K. Hoshiko, Q. Liu, T. Yagi, T. Nagayama, S. Kusaka, M. Tsuchiya, Y. Kitagawa, W.-Y. Wong, H. Nishihara, Nat. Comm. 2015, 6, 6713.

[44] P. Payamyar, K. Kaja, C. Ruiz-Vargas, A. Stemmer, D. J. Murray, C. J. Johnson, B. T. King, F. Schiffmann, J. VandeVondele, A. Renn, S. Götzinger, P. Ceroni, A. Schütz, L.-T. Lee, Z. Zheng, J. Sakamoto, A. D. Schlüter, Adv. Mater. 2014, 26, 2052.

[45] D. J. Murray, D. D. Patterson, P. Payamyar, R. Bhola, W. Song, M. Lackinger, A. D. Schlüter, B. T. King, J. Am. Chem. Soc. 2015, 137, 3450.

[46] V. Müller, A. Hinaut, M. Moradi, M. Baljozovic, T. A. Jung, P. Shahgaldian, H. Möhwald, G. Hofer, M. Kröger, B. T. King, E. Meyer, T. Glatzel, A. D. Schlüter, Angew. Chem. Int. Ed. 2018, 57, 10584.

[47] M. Matsumoto, L. Valentino, G. M. Stiehl, H. B. Balch, A. R. Corcos, F. Wang, D. C. Ralph, B. J. Mariñas, W. R. Dichtel, Chem. 2018, 4, 308.

[48] M. Zhao, Q. Lu, Q. Ma, H. Zhang, Small Meth. 2017, 1, 1600030.

[49] R. Dong, P. Han, H. Arora, M. Ballabio, M. Karakus, Z. Zhang, C. Shekhar, P. Adler, P. S. Petkov, A. Erbe, S. C. B. Mannsfeld, C. Felser, T. Heine, M. Bonn, X. Feng, E. Cánovas, Nat. Mater. 2018, 17, 1027.

[50] D. Zhou, X. Tan, H. Wu, L. Tian, M. Li, Angew. Chem. Int. Ed. 2019, 58, 1376.

[51] R. Matsuoka, R. Sakamoto, K. Hoshiko, S. Sasaki, H. Masunaga, K. Nagashio, H. Nishihara, J. Am. Chem. Soc. 2017, 139, 3145.

[52] S. Liu, F. Wang, R. Dong, T. Zhang, J. Zhang, X. Zhuang, Y. Mai, X. Feng, Adv. Mater. 2016, 28, 8365.

[53] M.-P. Pileni, Nat. Mater. 2003, 2, 145.

[54] S. Liu, J. Zhang, R. Dong, P. Gordiichuk, T. Zhang, X. Zhuang, Y. Mai, F. Liu, A. Herrmann, X. Feng, Angew. Chem. Int. Ed. 2016, 55, 12516. 